\title{
High-frequency two-input CMOS OTA for continuous-time filter applications
}

\author{
J.Glinianowicz, J.Jakusz, S.Szczepanski and Y.Sun
}

\begin{abstract}
A high-frequency fully differential CMOS operational transconductance amplifier (OTA) is presented for continuous-time filter applications in the megahertz range. The proposed design technique combines a linear cross-coupled quad input stage with an enhanced folded-cascode circuit to increase the output resistance of the amplifier. SPICE simulations show that DC-gain enhancement can be obtained without significant bandwidth limitation. The two-input OTA developed is used in high-frequency tuneable filter design based on IFLF and $L C$ ladder simulation structures. Simulated results of parameters and characteristics of the OTA and filters in a standard 1.2 $\mu \mathrm{m}$ CMOS process (MOSIS) are presented. A tuning circuit is also discussed.
\end{abstract}

\section{Introduction}

Continuous-time integrated filters have received substantial attention for high-frequency applications such as read channels in hard disc-drive systems, digital video, RF/IF filters, etc. Several integrated analogue filters with megahertz operation frequencies have been successfully implemented using the continuous-time transconductor-capacitor (Gm-C) technique [1-5].

The design of high-frequency $\mathrm{Gm}-\mathrm{C}$ filters requires highperformance OTAs. One of the standard techniques to improve the CMRR, PSRR and dynamic range of analogue integrated filters is to use fully differential structures $[6,7]$. Many OTAs suitable for such applications have been reported in recent years $[2-5,8-10]$.

The integrator is the main building block of active filters. One of the major problems in high-frequency applications is the phase error of the integrator $[4,9]$. To keep the phase as close as possible to $-90^{\circ}$, a wideband OTA with sufficiently high $\mathrm{DC}$ gain is required. If this can be achieved the $Q$-tuning circuit for filters will not be required.

In this paper a novel CMOS fully differential OTA with high DC gain and wide bandwidth is presented. The twoinput structure is especially useful in filter design [1, 10-12], allowing to reduce the number of active components and the chip area. The proposed circuit was designed and simulated using a standard 1.2 $\mathrm{m}$ AMI ABN CMOS process. Application examples of filter design are also given together with a tuning system.

\footnotetext{
(C) IEE, 2000

IEE Proceedings online no. 20000317

DOI: $10.1049 /$ /p-cds:20000317

Paper first received 25th June 1999 and in revised form 28th January 2000

J. Glinianowicz, J. Jakusz and S. Szczepanski are with the Faculty of Electronics, Telecommunications and Informatics, Technical University of Gdansk, 80 952 Gdansk, Poland

Y. Sun is with the Department of Electronic, Communication and Electrical Engineering, University of Hertfordshire, Hatfield AL10 9AB, UK
}

\section{Two-input OTA circuit description}

The proposed design technique combines the cross-coupled quad input stage $[5,8-10]$ with the enhanced foldedcascode circuit [13-15] to increase the output resistance of the amplifier. The input stage consisting of transistors Mla-M4a and M1b-M4b in Fig. 1 is in fact a linear $V-I$ converter. Using the standard square-law model for MOS transistors in their saturation region, the differential output current $I_{\text {out }}$ can be expressed as

$$
I_{\text {out }}=k_{n} V_{B}\left\lfloor\left(V_{i a+}-V_{i a-}\right)+\left(V_{i b+}-V_{i b-}\right)\right\rfloor
$$

where $k_{n}=0.5 \mu_{o} C_{o x}(W / L)$ is the transconductance parameter $\left(\mu_{o}, C_{o x}, W\right.$ and $L$ are the mobility, oxide capacitance per unit area, and channel width and length, respectively), $V_{B}$ is the voltage of the floating voltage source connected between points $\mathrm{C}_{\mathrm{R} 1}$ and $\mathrm{C}_{\mathrm{R} 2}$, and $V_{i d a}=\left(V_{i a+}-V_{i a-}\right), V_{i d b}$ $=\left(V_{i b+}-V_{i b-}\right)$ are the differential input voltages. It was assumed that the transistors Mla-M4a and M1b-M4b have the same dimensions $W$ and $L$. This circuit can be considered as two identical transconductance stages with output nodes connected in parallel and exhibits a perfectly linear transconductance of value $g_{m}=k_{n} V_{B}$. The $V-I$ converter is tuneable by varying bias voltage $V_{B}$, its detailed analysis being reported in [8,9]. As shown in Fig. 2 the control of the voltage $V_{B}$ is achieved by varying the direct current $I_{C F}$. A similar floating voltage source of low output resistance was also described in $[5,8,9]$.

The output stage employs an enhanced folded-cascode circuit and is composed of transistors M5-M12. Transistors MI17 and MI18 form the improved cascode current mirror. Since negative current-shunt feedback is used, the output resistance looking into the drains of $\mathrm{M} 5 / \mathrm{M} 6$ is increased. Applying small-signal analysis and neglecting the body effect, the simplified expression for one of the two identical branches is given as

$$
\begin{aligned}
r_{d 5}=r_{d 6}= & \left\lfloor r_{d 9} \| r_{V-I}+r_{d s 5}\left(1+g_{m 5} r_{d 9} \| r_{V-I}\right)\right\rfloor \\
& \times\left(1+g_{m 7} r_{d s 7}\right)
\end{aligned}
$$

where $r_{d 9}=r_{d s 11}+r_{d s 9}\left(1+g_{m 9} r_{d s 11}\right)$ is the output resistance of cascodes M9, M11 and M10, M12, and $r_{V-I}$ is the 


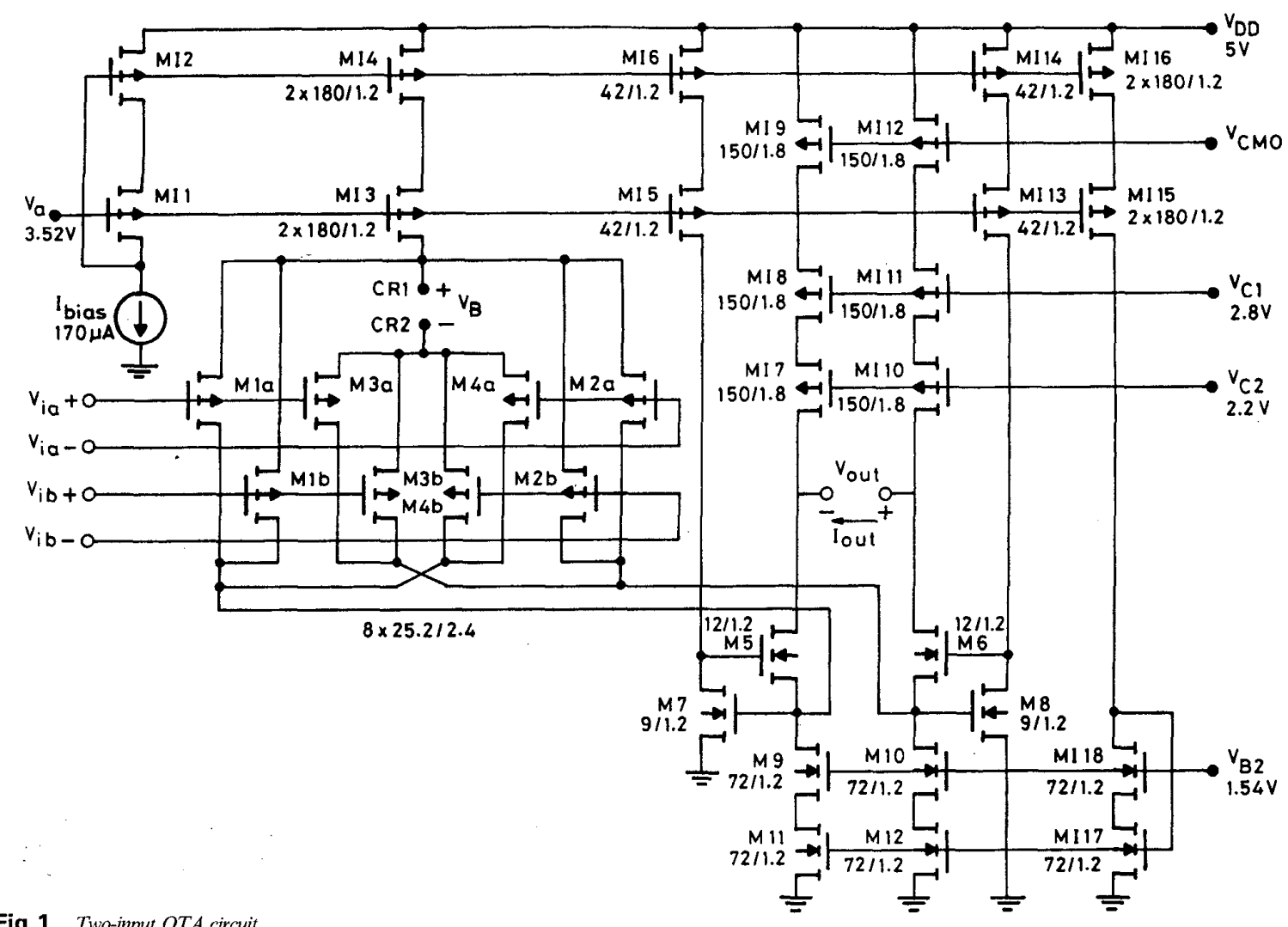

Fig. 1 Two-input OTA circuit

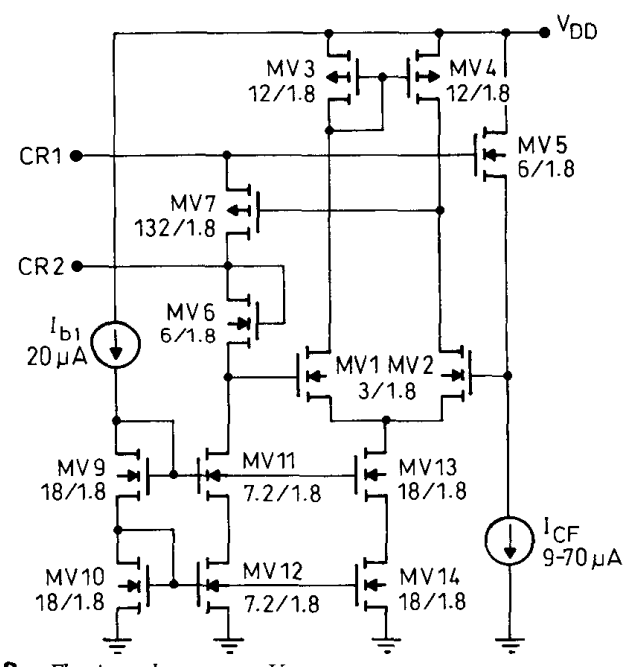

Fig.2 Floating voltage source $V_{B}$

As shown in [15], DC gain enhancement by current feedback in the output cascode has no significant effect on the high-frequency response. The common-mode voltage at the output nodes is stabilised by the typical common-mode feedback loop shown in Fig. 3 [7]. The desired value is $2.5 \mathrm{~V}$, i.e. a half of the power supply voltage $V_{D D}$.

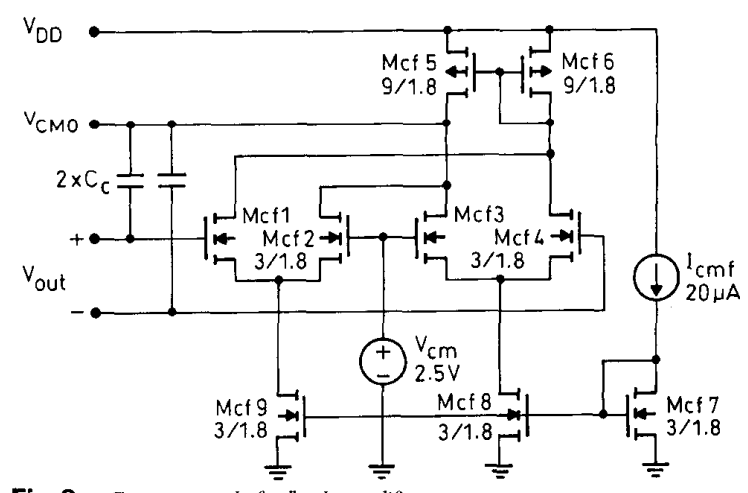

Fig.3 Common-mode feedback amplifier

parallel connection of drain-source resistance $r_{d s}$ of the transistors M1a, M1b, M4a, M4b or M2a, M2b, M3a, $\mathrm{M} 3 \mathrm{~b}$. To calculate the overall differential output resistance $r_{\text {out }}$ of the OTA in Fig. 1, the connection of p-channel transistors MI7, MI8, MI9, MI10, MI11 and MI12 forming a tripled-cascode circuit has to be taken into account. As a result, the output resistance $r_{\text {out }}$ can be written as

$$
\begin{aligned}
r_{\text {out }}=2 \cdot\{ & r_{d 5} \|\left[r_{d s I 7}+\left(1+g_{m I 7} r_{d s I 7}\right)\right. \\
& \left.\left.\times\left(r_{d s I 8}+r_{d s I 9}+g_{m I 8} r_{d s I 8} r_{d s I 9}\right)\right]\right\}
\end{aligned}
$$

Thus the DC gain for both input pairs is determined as

$$
A_{d D C}=g_{m} r_{\text {out }}
$$

\section{Second-order effects}

Mobility reduction, body effects and channel length modulation can cause distortion in the linear transfer function in eqn. 1. An analysis of the influence of second-order effects on a similar transconductance stage has been reported in [9]. The input stage remains linear if the following scaling condition is fulfilled:

$$
\frac{K_{1 a, 1 b, 2 a, 2 b}}{K_{3 a, 3 b, 4 a, 4 b}}=\frac{\left[\theta\left(V_{q}+V_{T 1 a, 1 b, 2 a, 2 b}\right)-1\right]^{4}}{\left[\theta\left(V_{q}+V_{B}+V_{T 3 a, 3 b, 4 a, 4 b}\right)-1\right]^{4}}
$$


where

$$
\begin{aligned}
& \theta=\frac{1}{T_{O X} E_{C R}} \\
& V_{T n}=V_{T o}+\gamma\left(\sqrt{\Phi-V_{B S n}}-\sqrt{\Phi}\right) \\
& V_{q}=-\frac{V_{P}+V_{Q}}{2}
\end{aligned}
$$

where $K_{1 a}$ to $K_{4 b}$ are the transconductance parameters of transistors Mla to M4b, $\theta$ is the coefficient of the effect of the electric field on the mobility, $\mathrm{T}_{O X}$ is the gate oxide thickness, $E_{C R}$ is the critical field, $V_{B S n}$ is the bulk-source voltage of transistor $\mathrm{M}_{n}, V_{T n}$ is the threshold voltage, $V_{T_{0}}$ is the threshold voltage for $V_{B S}=0, \gamma$ is the bulk threshold parameter, $\Phi$ is the strong inversion surface potential, and $V_{P}$ and $V_{Q}$ are the source-gate voltages of Mla (M1b) and M2a (M2b), respectively. Because the condition in eqn. 5 is a function of tuning voltage $V_{B}$, the scaling is possible only for a given transconductance $g_{m}$ of the OTA.

The channel length modulation effect of transistors Mla to M4b can be neglected owing to the application of current-shunt feedback in the output stage.

\section{Simulation results}

The OTA was simulated using SPICE level 3 transistor models for $1.2 \mu \mathrm{m}$ AMI ABN CMOS process. The bias current flowing through the input stage is equal to $340 \mu \mathrm{A}$. All p- and n-channel transistors have their bulks connected to $V_{D D}$ and GND, respectively. The main parameters of the OTA obtained from the simulation are presented in Table 1. As can be seen, the transconductance may be tuned by a decade. Implementation of the enhanced folded cascode in the output stage results in an increase of output resistance by 11 times, which increases the DC voltage gain significantly. The worst-case PSRR and worst-case CMRR were simulated assuming equal $1 \%$ mismatches in all transconductance parameters $k_{n}$ and $k_{p}$, as well as in all threshold voltages $V_{T n}$ and $V_{T p}$ of MOS devices.

Table 1: Simulated results of OTA

\begin{tabular}{ll}
\hline Parameter & Value @ $V_{D D}=5 \mathrm{~V}$ \\
\hline Transconductance range & $5-50 \mu S$ for $I_{C F} 9-70 \mu \mathrm{A}$ \\
DC voltage gain & $50-70 \mathrm{~dB}$ for $I_{C F} 9-70 \mu \mathrm{A}$ \\
THD at 10MHz, with $V_{\text {id }}=0.5 \mathrm{~V}$ (magnitude) & $<-47.1 \mathrm{~dB}$ for $I_{C F} 9-70 \mu \mathrm{A}$ \\
CMRR & $>46 \mathrm{~dB}$ \\
PSRR & $>62 \mathrm{~dB}$ \\
Equivalent input capacitance $C_{\text {in }}$ & $0.048 \mathrm{pF}$ \\
Equivalent output capacitance $C_{\text {out }}$ & $0.033 \mathrm{pF}$ \\
Power consumption & $7.65 \mathrm{~mW}$ \\
\hline
\end{tabular}

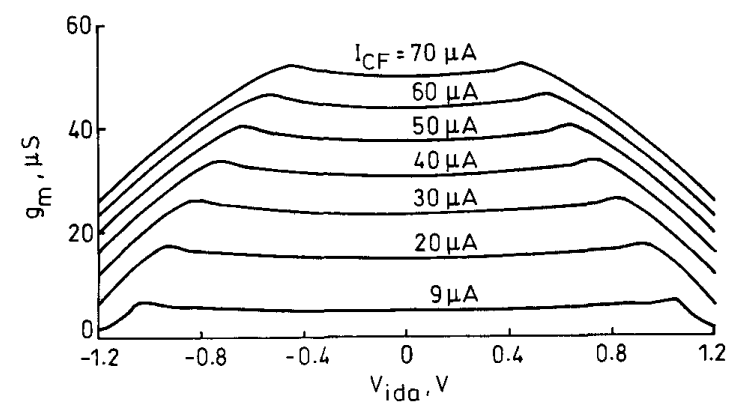

Fig.4 Transconductance tuning capability of complete OTA at $V_{i d b}=0$
The simulated transconductance $g m$ as a function of tuning current $I_{C F}$ and input voltage $V_{i d a}$ is presented in Fig. 4. The differential voltage $V_{i d b}$ is assumed to be equal to zero. Similarly, identical characteristics can be obtained for the function of $g_{m}$ against $I_{C F}$ and $V_{i d b}$ with $V_{i d a}=0$. Connecting the two input pairs together doubles the transconductance achieved. As can be seen from Fig. 4 the allowed input voltage amplitude range is limited to $0.5 \mathrm{~V}$.

Fig. 5 shows the OTA short-circuit frequency response. The 3-dB frequency is placed in the $\mathrm{GHz}$ region so the OTA can be applied to high-frequency signal processing. The Gm-C integrator was simulated with the load capacitance of value $1 \mathrm{pF}$ connected differentially. The results are given in Fig. 6. The maximum frequency of the integrator at which the phase error is less then 1 degree, is about $85 \mathrm{MHz}$. The very high DC gain is another advantage which makes the circuit very useful in systems without $Q$-tuning.

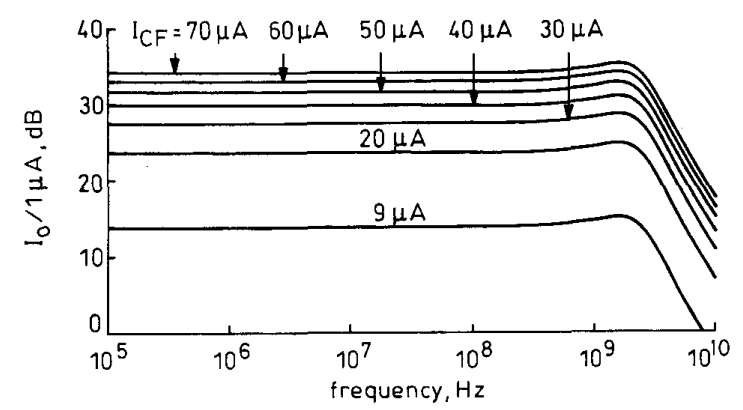

Fig.5 Simulated frequency response of OTA normalised to $I \mu A$

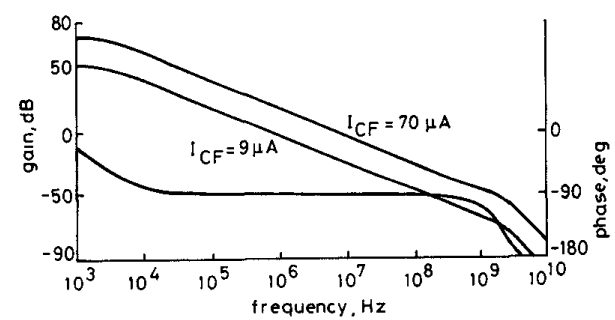

Fig. 6 Simulated gain and phase responses of $\mathrm{Gm}-\mathrm{C}$ integrator

\section{$5 \quad$ Filter examples}

A tuneable $40 \mathrm{MHz}$ third-order elliptic filter was designed and simulated using the OTA. The normalised passive prototype and the corresponding active implementation are given in Figs. 7 and 8, respectively. The input OTA has two times the transconductance to compensate for the $6 \mathrm{~dB}$ loss in passband. It can be seen that in every node two or four OTA stages share a capacitor. A realisation based on twoinput OTAs results in the number of active elements two times lower.

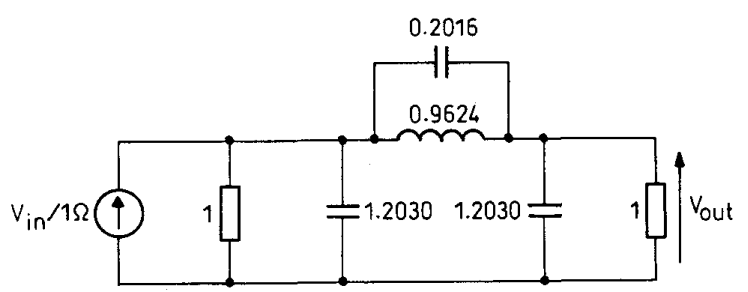

Fig.7 RLC prototype of third-order elliptic filter 


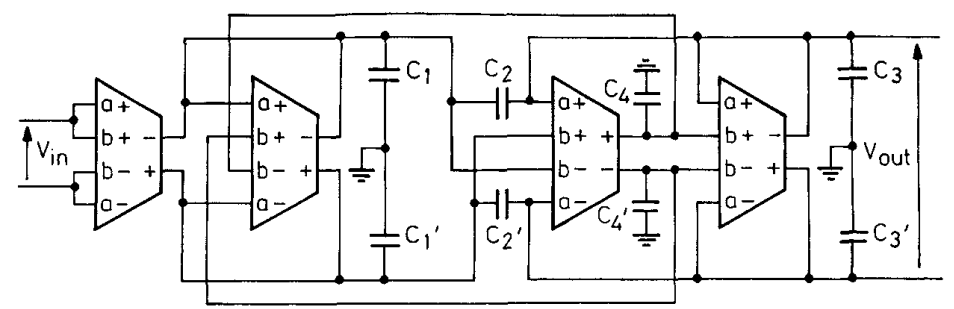

Fig.8 Low-pass active implementation of third-order elliptic filter based on two-input OTA

Having in mind the accuracy of filter characteristics and the chip area, both extra and parasitic capacitances must be taken into account while designing a filter in the megahertz range. Fortunately in the filter structure considered, all input and output OTA capacitances and some extra capacitances sum which gives the following values of elements:

$$
\begin{aligned}
& C_{1}=C_{1}^{\prime}=638 \mathrm{fF}-2\left(2 C_{\text {in }}+2 C_{\text {out }}\right)=314 \mathrm{fF} \\
& C_{3}=C_{3}^{\prime}=638 \mathrm{fF}-2\left(2 C_{\text {in }}+C_{\text {out }}\right)=380 \mathrm{fF} \\
& C_{4}=C_{4}^{\prime}=511 \mathrm{fF}-2\left(2 C_{\text {in }}+C_{\text {out }}\right)=253 \mathrm{fF} \\
& C_{2}=C_{2}^{\prime}=115 \mathrm{fF} .
\end{aligned}
$$

Although the filter is designed to a particular frequency, it is possible to tune the cut-off frequency by varying $I_{C F}$ in a wide range from 4 to $40 \mathrm{MHz}$. High attenuation at transmission zeros is achieved owing to the excellent OTA output resistance, Fig. 9. An attenuation of the $R L C$ prototype in the stopband of $28 \mathrm{~dB}$ is achieved in active implementation.

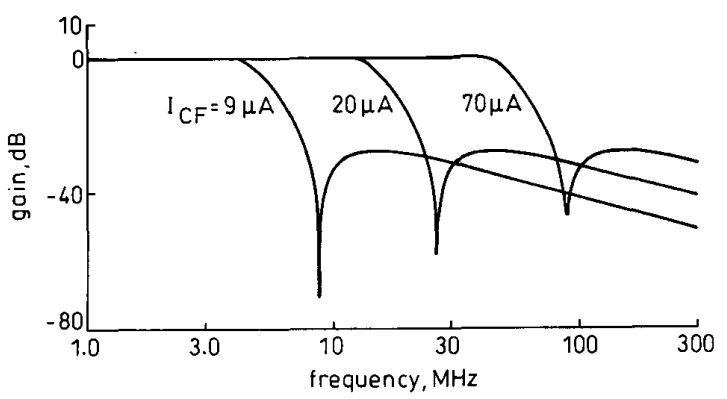

Fig.9 Simulated amplitude responses of elliptic filter in Fig. 8

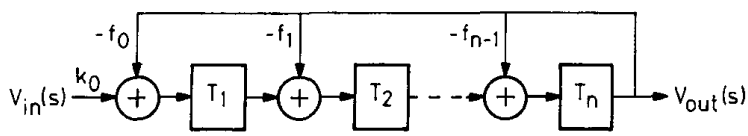

Fig. 10 Block diagram of IFLF low-pass filter

The two-input OTA has also been used to design a seventh-order IFLF low-pass filter. The topology of the filter is presented in Fig. 10. $T_{j}$ blocks are either integrators or biquad circuits and $f_{j}$ are feedback coefficients which determine the denominator of the transfer function. In general, there is a simple way to introduce gain-boost real-axis zeros or any desired transmission zeros to the transfer function by adding feedforward branches from the input to the summing nodes $[1,10-12]$.

The designed filter is based on the diagram in Fig. 11. Symbols $2 x$ and $4 \times$ mean 2 or 4 unit OTAs connected in parallel. The cut-off frequency has been chosen to be $4 \mathrm{MHz}$. With transconductance equal to $50 \mu \mathrm{S}$ at $I_{C F}=$ $70 \mu \mathrm{A}$, the following values of capacitors can be obtained:

$C_{1}=C_{1}^{\prime}=12.64 \mathrm{pF}-2\left(C_{\text {out }}+C_{\text {in }}\right)=12.48 \mathrm{pF}$

$C_{2}=C_{2}^{\prime}=5.86 \mathrm{pF}-2\left(C_{\text {out }}+C_{\text {in }}\right)=5.7 \mathrm{pF}$

$C_{3}=C_{3}^{\prime}=3.62 \mathrm{pF}-2\left(C_{\text {out }}+C_{\text {in }}\right)=3.46 \mathrm{pF}$

$C_{4}=C_{4}{ }^{\prime}=2.39 \mathrm{pF}-2\left(C_{\text {out }}+C_{\text {in }}\right)=2.23 \mathrm{pF}$

$C_{5}=C_{5}^{\prime}=1.71 \mathrm{pF}-2\left(C_{\text {out }}+2 C_{\text {in }}\right)=1.45 \mathrm{pF}$

$C_{6}=C_{6}^{\prime}=2.12 \mathrm{pF}-2\left(2 C_{\text {out }}+4 C_{\text {in }}\right)=1.6 \mathrm{pF}$

$C_{7}=C_{7}^{\prime}=3.04 \mathrm{pF}-2\left(4 C_{\text {out }}+11 C_{\text {in }}\right)=1.72 \mathrm{pF}$.

In the range of bias current $I_{C F}$ of the OTAs the cut-off frequency of the filter varies ten times, as can be seen from Fig. 12.

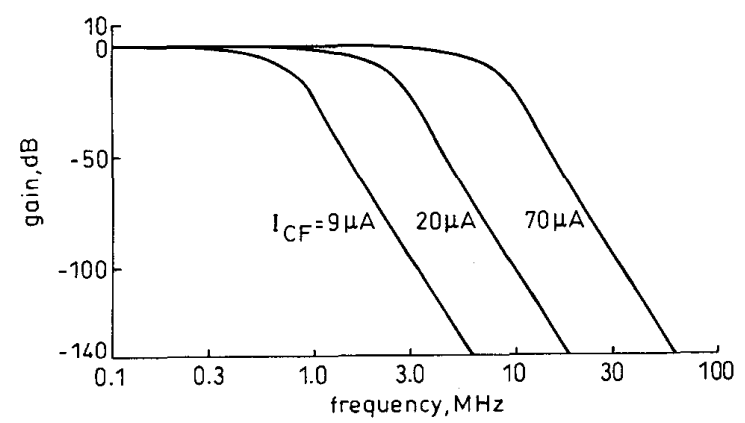
Fig. 12 Simulated frequency response of seventh-order IFLF low-pass filter
in Fig. 11

\section{Tuning system}

Owing to process parameter variations, thermal effects and mismatches the tuning system is of great importance. The most common way to tune a continuous-time $\mathrm{Gm}-\mathrm{C}$ circuit is a master-slave structure $[3,4]$. This approach assumes a good match between the master and slave OTAs. The proposed tuning circuit consists of blocks shown in Fig. 13. This is a typical phase-locked loop (PLL) with an on-chip loop filter, thus no external components are required. The

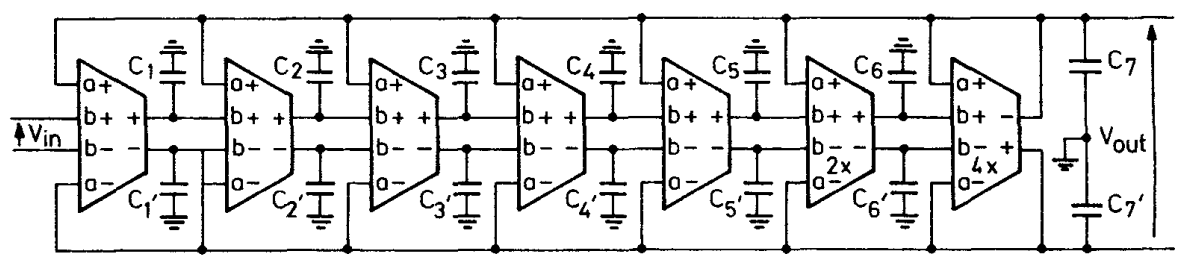

Fig.11 Seventh-order IFLF low-pass filter based on two-input OTA 


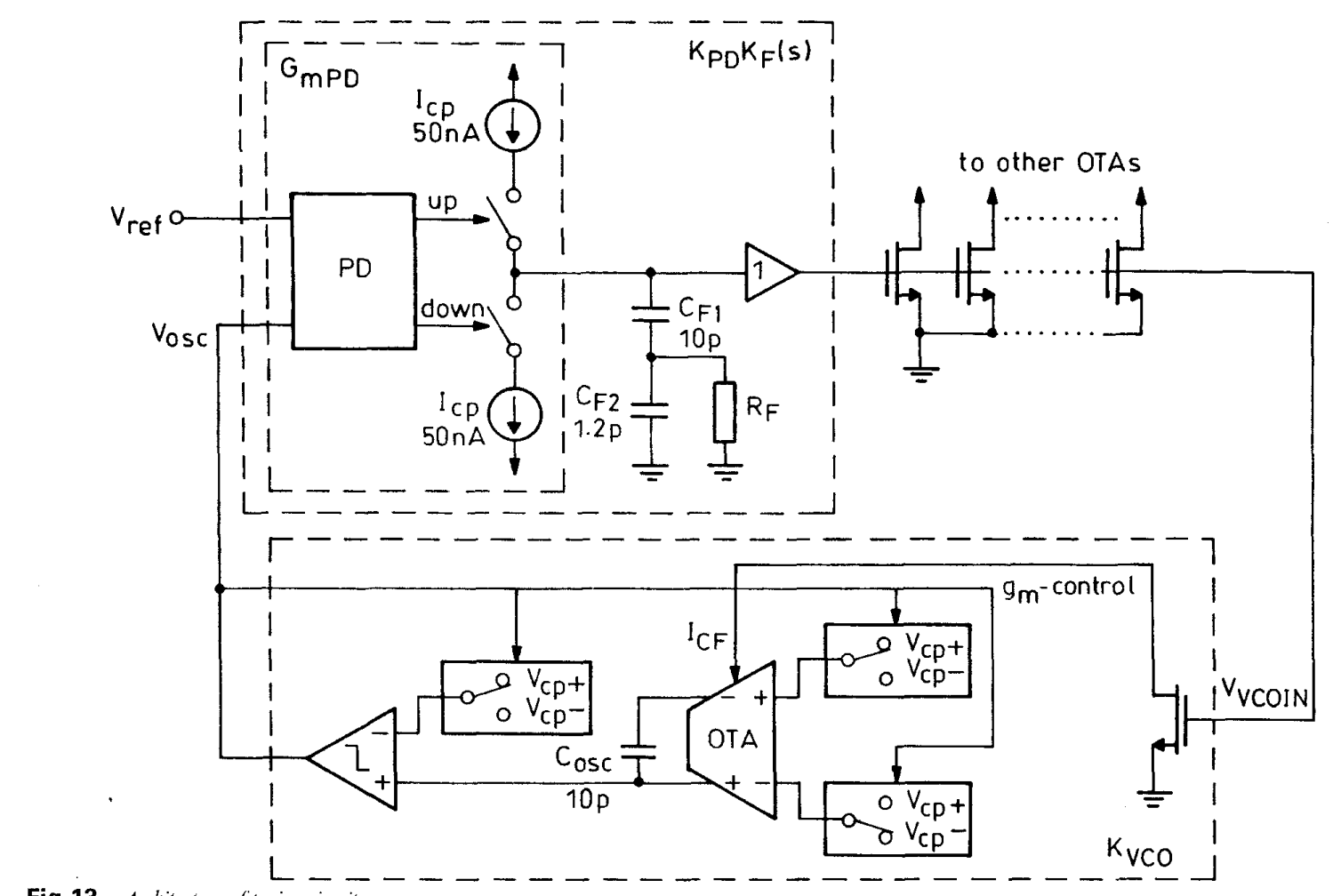

Fig. 13 Architecture of tuning circuit

open-loop transfer function of the PLL is written as

$$
A(s)=K_{P D} \cdot K_{F}(s) \cdot \frac{K_{V C O}}{s}
$$

where $K_{P D}$ is the phase comparator gain, $K_{F}(s)$ represents the low-pass filter transfer function and $K_{V C O}$ is the oscillator gain. $K_{V C O}$ is divided by $s$ because the frequency of the VCO output is converted to phase at the input of the phase comparator.

The phase detector subcircuit is in fact a phase-frequency one with two edge-triggered D-type flip-flops and a three-state output stage. By using current sources as the charge pump the dead zone could be reduced to zero. The PLL loop filter is of the lag-lead type, where the charge pump works as an active part. The transfer function of this block can be expressed as

$$
\begin{aligned}
K_{P D} \cdot K_{F}(s) & =\frac{1+s R_{F}\left(C_{F 1}+C_{F 2}\right)}{s \frac{C_{F 1}}{G_{m P D}}\left(1+s R_{F} C_{F 2}\right)} \\
& =\frac{1+s \tau_{2}}{s \tau_{1}\left(1+s \tau_{3}\right)}
\end{aligned}
$$

$G_{m P D}$ is the transconductance of the charge pump, which is equal to $I_{C P} / 2 \pi$, i.e. $8 \mathrm{nS}$. A simple $R C$ filter can result in a very small phase margin. If a zero associated with time constant $\tau_{2}$ is introduced to the filter the phase margin of the PLL can be increased to a desired value, for example, $60^{\circ}$, avoiding instability. Adding $C_{F 2}$ improves the attenuation of the current spikes from the phase detector. Since a typical ratio of $C_{F 2} / C_{F 1}$ is about $0.1, C_{F 1}$ can be realised as a floating capacitor between poly1 and poly2, and $C_{F 2}$ as a parasitic one to the substrate. $R_{F}$ represents the resistance equivalent to the connection of several transistors polarised by a certain current. The lock range for this type of phase detector is equal to the capture range and is independent of the low-pass filter.
The connection of the tuneable Gm-C integrator based on the same OTA as those inside the filter and the comparator with hysteresis builds the VCO. Reference voltages $V_{c p+}$ and $V_{c p-}$ are generated internally. Increasing or decreasing the slope of the triangle voltage at the integrator's output can compensate for the changes of comparator's trip voltages with power supply. The frequency of the $\mathrm{VCO}$ is determined by

$$
f=\frac{g_{m}\left(I_{C F}\right)}{2 C_{O S C}}=\frac{g_{m}\left(V_{V C O I N}\right)}{2 C_{O S C}}
$$

which is independent of trip and reference voltages $V_{c p+}$ and $V_{c p}$. The transconductance $g_{m}()$ of the OTA is tuned simultaneously with slave OTAs (forming the core of the IC) by the voltage from the phase detector/loop filter block.

\section{Conclusions}

A fully differential two-input CMOS OTA suitable for high-frequency analogue filter applications has been presented. The use of the two-input OTA in filter design has been illustrated, which significantly reduces the number of active elements and simplifies the structure. A tuning scheme has also been discussed. Simulation results have shown good performance of the developed CMOS OTA and its filtering applications in megahertz range. The proposed OTA can be easily expanded to have more differential inputs simply by adding more cross-coupled stages M1 $x-M 4 x$ connected in parallel with existing ones.

\section{Acknowledgment}

This work was supported by the Polish State Scientific Research Committee under grant 8T11B01114. Part of the work was done during Stanislaw Szczepanski's visit at the University of Hertfordshire in 1998. 


\section{References}

1 DELIYANNIS, T., SUN, Y., and FIDLER, J.K.: 'Continuous-time active filter design' (CRC Press, USA, 1999)

2 ALINII, R., BASCHIROTTO, A., and CASTELLO, R.: 'Tuneable BiCMOS continuous-time filter for high-frequency applications', IEEE J. Solid-State Circuits, 1992, 27, (12), pp. 1905-1915

3 SILVA-MARTINEZ, J., STEYAERT, M.S.J., and SANSEN, W.: 'A 10.7-MHz 68-dB CMOS continuous-time filter with on-chip automating tuning', IEEE J. Solid-State Circuits, 1992, 27, (12), pp. 1843-1853

4 NAUTA, B.: 'Analog CMOS filters for very high frequencies' (Kluwer Academic Publishers, 1993)

5 PANKIEWICZ, B., JAKUSZ, J., and SZCZEPANSKI, S.: 'A $27 \mathrm{MHz}$ fully-balanced OTA-C filter in $2 \mu \mathrm{m}$ CMOS technology' in NAPIERALSKI, A., CIOTA, Z., MARTINEZ, A., DE MEY, G. and CABESTANY, J. (Eds.): 'Mixed design of integrated circuits and systems' (Kluwer Academic Publishers, 1998), pp.35-40

6 STEYAERT, M.S.J., and SANSEN, W.: 'Power supply rejection ratio in operational transconductance amplifiers', IEEE Trans. Circuits Syst., 1990, 37, (9), pp. 1077-1084

7 VANPETEGHEM, P.M., and DUQUE-CARRILLO, J.F.: 'A general description of common-mode feedback in fully-differential amplifiers'. Proceedings of IEEE international symposium on Circuits and systems, 1990, pp. 3209-3212
8 SZCZEPANSKI, S., JAKUSZ, J., and SCHAUMANN, R.: 'A linear CMOS OTA for VHF applications'. Proceedings of IEEE international symposium on Circuits and systems, 1995, Vol. 2, pp. 1344-1347

9 SZCZEPANSKI, S., JAKUSZ, J., and SCHAUMANN, R.: 'A linear fully balanced CMOS OTA for VHF filtering applications', IEEE Trans. Circuits Syst. II, Analog Digit. Signal Process., 1997, 44, (3), pp. Trans. Circe

10 CHIANG, D.H., and SCHAUMANN, R.: 'A CMOS fully-balanced continuous-time IFLF filter design for read/write channels'. Proceedings of IEEE international symposium on Circuits and systems, 1996 , Vol. 1, pp. 167-170

11 SUN, Y., and FIDLER, J.K.: 'OTA-C realisations of general highorder transfer functions', Electron. Lett., 1993, 29, (12), pp. 1057-1058

12 SUN, Y., and FIDLER, J.K.: 'Synthesis and performance analysis of universal minimum component integrator-based IFLF OTA-grounded capacitor filter', IEE Proc., Circuits, Devices Syst., 1996, 143, (2), pp. $107-114$

13 SACKINGER, E., and GUGENBUHL, W.: 'A high-swing, highimpedance MOS cascode circuits', IEEE J. Solid-State Circuits, 1990, 25, pp. 289-298

14 YANG, H.C. and ALLSTOT, D.J.: 'An active-feedback cascode current source', IEEE Trans. Circuits Syst., 1990, 37, (5), pp. 644646

15 ZARABADIL, S.R., and ISMAIL, M.: 'Very-high-output-impedance cascode current sources/current mirrors/transresistance stages and their applications', Int. J. Circuit Theory Appl., 1992, 20, pp. 639-648 